\title{
From End-User's Requirements to Web Services Retrieval: a Semantic and Intention-Driven Approach
}

\author{
Isabelle Mirbel and Pierre Crescenzo \\ Université de Nice Sophia-Antipolis \\ Laboratoire I3S (UNS/CNRS) \\ 930 route des Colles \\ BP 145 \\ F-06903 Sophia-Antipolis cedex \\ France \\ \{Isabelle.Mirbel, Pierre.Crescenzo\}@unice.fr
}

Summary. In this paper, we present SATIS, a framework to derive Web Service specifications from end-user's requirements in order to operationalise business processes in the context of a specific application domain. The aim of SATIS is to provide to neuroscientists, which are not familiar with computer science, a complete solution to easily find a set of Web Services to implement an image processing pipeline. More precisely, our framework offers the capability to capture high-level end-user's requirements in an iterative and incremental way and to turn them into queries to retrieve Web Services description. The whole framework relies on reusable and combinable elements which can be shared out by a community of users sharing some interest or problems for a given topic. In our approach, we adopt Web semantic languages and models as a unified framework to deal with end-user's requirements and Web Service descriptions in order to take advantage of their reasoning and traceability capabilities.

Key words: Web Services; Semantic Web; Intentional Modeling; Rules; Reuse

\subsection{Introduction}

Service-oriented computing is a paradigm relying on services as atomic constructs to support the development and easy composition of distributed applications. Application components are assembled with little efforts into workflows of services loosely coupled to operationalise flexible and dynamic business processes. Searching for the relevant Web Services to operationalise a particular business process is one of the challenges of the service-oriented computing area. At present, in the process of searching for Web Services, it is assumed that user's goals have already been identified, captured, specified 
and formalised in a suitable model to easily find the relevant services. Or it is considered that users, which often are specialists of their domain, are also computer scientists or at least connoisseurs of Web Services. These hypotheses are generally too strong to be reasonable.

In this context, the SATIS (Semantically AnnotaTed Intentions for Services) project's ambition is to allow final users to express their intentions (or goals) and strategies (to achieve their intentions) in a high-level language, and to support the selection of a set of Web Service descriptions which could respond to the users' needs. But this problem is complex and cannot be solved in a general approach. Therefore, we focus on an application domain where domain knowledge and service descriptions (semantic Web Services) are available. The aim of SATIS is to provide to neuroscientists, which are not familiar with computer science, a complete solution to easily find a set of Web Services to implement an image processing pipeline.

Indeed, our purpose is to give at users disposal some useful dedicated reusable fragments of know-how to help them to implement their business goals with Web Services. Therefore, our approach relies on high-level businessoriented activity specification with the help of an intentional model in order to derive Web Service description from this high-level specification. We also focus on a community of users sharing some interest or problems for a given topic inside the business domain.

Our work belongs to the family of goal-based service retrieval approaches. These approaches $(23,24,25,2])$ aim at specifying the goals which have to be satisfied by the retrieved services. In these proposals, different models are provided to specify goals without addressing the problem of how to capture them. On the contrary, our aim is to provide means to assist final users in querying the Web Service registry to find Web Services to operationalise a business process. The GODO approach [8] also addresses this issue by proposing models and tools to capture user's goals with the help of an ontology or in natural language. As in [10, we propose an incremental process to refine users' requirements in order to specify the features required for the Web Services under retrieval. Our approach distinguishes itself from [10 by the fact that we rely on semantic Web models and techniques to enrich the goal (or intention) specification, in order to provide reasoning and explanation capabilities.

With regards to approaches dealing with ontology-based service discovery 12. and more precisely OWL-S based approaches (as we are relying on OWL$\mathrm{S}$ with regards to Web Service descriptions), capability matching algorithms [11 exploiting service profile descriptions have been proposed. Matchmaking algorithms [15] comparing state transformations described in the query to the ones provided in the descriptions have also been proposed. All these algorithms mainly exploit features of subsumption relationships. Ranking mechanisms have also been provided 11. Our approach distinguishes itself from these works by the fact that our focus is on providing means to assist final users in authoring queries (more than rendering them). In other words, we are interested in the upstream process of deriving queries from final users re- 
quirements. Moreover, our concern is also on how to annotate such queries in order to support their capitalisation and sharing among a community of users.

Beyond an alternative way to search for Web Services, we provide means to capitalise know-how about Web Service search procedures themselves. Another novelty of our approach is to operationalise goals by rules in order to promote both mutualisation of high-level intentional specification and crossfertilisation of know-how about Web Services search procedures among the community members.

The paper is organised as follows. First we give an overview of our SATIS approach in section 1.2. Then, in section 1.3, we detail the authoring process proposed in SATIS and how the authored search procedure is rendered in section 1.4 Next, we explain in section 1.5 how the framework is used by the different actors interacting in a neurosciences community of users. Finally, we conclude and give some perspectives.

\subsection{SATIS approach}

The aim of our approach is to provide to neuroscientists, which are not familiar with computer science, a complete solution to easily find a set of Web Services to implement an image processing pipeline. The focus of this proposal is on how to search and retrieve Web Services descriptions from end-user's requirements. Indeed we provide support to retrieve an organised set of Web Service descriptions suitable to operationalise an image processing pipeline as specified by a neuroscientist (final user).

As we are interested by high-level end-user's requirements, we rely on a dedicated graphical notation to capture and specify them. In the context of a neuroscientists community, these requirements deal with image analysis pipelines. Different business process modeling formalisms have been proposed in the literature [13. Decision-oriented models are semantically more powerful than the other process models because they explain not only how the process proceeds but also why. Their enactment guides the decision making process that shapes the process, and helps reasoning about the rationale [13. Our approach is based on the adaptation of such a decision-oriented model called the map model [21]. This intentional process modeling formalism allows final users (neuroscientists) to define their image analysis pipeline by describing intermediate intentions (i.e. goals and subgoals to be satisfied through the processing chain) and strategies (i.e. means to reach goals).

As we are interested by the end-user's point of view on the processing pipeline to be operationalised by Web Services, we don't want him/her to explicitly specify the Web Service(s) s/he is interested in but the intention(s) s/he wants to satisfy by rendering Web Service(s). Moreover, we don't want to explicitly associate Web Service descriptions to high-level end-user's intentional requirements. In our framework, end-users associate queries to their 
requirements. Indeed, queries allow end-users to specify generic Web Service descriptions. For instance, in a neuroscientist community, by looking for a Web Service which takes as input an image and provides as output a debiased image, the end-user specifies the kind of Web Service s/he is interested in without explicitly refering to one specific Web Service. By doing so, we assume a loosely coupling between high-level end-user's intentional requirements on one hand and Web Services descriptions on the other hand: if new Web Service descriptions are added inside the community Web Service registry, they can be retrieved to operationalise a high-level end-user's intentional requirement even if the requirement has been specified before the availability of the Web Services under consideration; and if Web Service descriptions are removed from the community Web Service registry, the high-level end-user's intentional requirements that they satisfied are still valid and may be operationalised by other available Web Services. Web Services are dynamically selected when rendering queries associated to high-level end-user's intentional requirements.

In our approach, we also adopt Web semantic languages and models as a unified framework to deal with (i) high-level end-user's intentional requirements, (ii) generic Web Service descriptions and (iii) Web Service descriptions themselves. With regards to high-level end-user's intentional requirements, we adapted the map model 21 to our concern and gathered its concepts and relationships into an RDFS [20] ontology dedicated to the representation of intentional processes: the map ontology [5]. As a result, intentional processes annotated with concepts and relationships from this ontology can be shared and exploited by reasoning on their representations. We also consider semantic Web Service descriptions specified with the help of the OWL-S ontology 14. And finally, generic Web Service descriptions are specified with the help of the W3C standard query language for RDF [19] annotations: SPARQL [22]. Generic Web Service descriptions are formalised into graph patterns over Web Services descriptions. Indeed, our approach relies on three ontologies: The map ontology we proposed 5 , the OWL-S ontology 14 and a domain ontology (in our case an ontology describing medical images and medical image processing dedicated to the neuroscience domain).

Knowledge capitalisation, management and dissemination inside a community of members may be supported by a collective memory, that is to say an explicit, disembodied and persistent representation of the community knowledge in order to facilitate access, sharing and reuse [6. In semantic collective memories, resources are indexed by semantic annotations in order to explicit and formalise their informative content. Information retrieval inside the collective memory relies on the formal manipulation of these annotations and is guided by ontologies. In SATIS, we are dealing with annotations about Web Service descriptions, generic Web Service descriptions and high-level end-user's intentional requirements. We are exploiting reasoning and traceability capabilities of semantic Web models and languages to provide dedicated search, sharing and reuse means to improve collaboration inside a community of neuroscien- 
tists. Beyond a way to retrieve Web Services, our approach aims at providing means to promote mutualisation of high-level end-user's intentional requirements and cross fertilisation of know-how about how to operationalise image processing pipelines among the community members. Our proposal may be compared to case based reasoning approaches in that it provides means to identify relevant Web Service descriptions (solutions) corresponding to new high-level end-user's intentional requirements (problems) based on Web Service descriptions (solutions) identified for similar requirements (problems). Indeed high-level end-user's intentional requirements are considered as problem descriptions and Web Service descriptions are considered as solutions. generic Web Service descriptions as well as subgoals and strategies elicited to specify high-level end-user's intentional requirements are considered as intermediary knowledge on which to reason to reduce the gap between high-level end-user's intentional requirements and Web Service descriptions thus providing solutions to problems that is to say proposing Web Services to implement an image processing pipeline.

Indeed, we address the issue about how to retrieve Web Service descriptions from high-level end-user's intentional requirements by providing means to reuse existing knowledge about relevant Web Services to operationalise high-level end-user's requirements inside the scope of a community of users.

\subsection{SATIS authoring process}

In SATIS, search procedure authoring is supported by a three steps process: (i) high-level end-user's intentional requirements elicitation, (ii) requirements and generic Web Service description formalisation and (iii) fragment definition. During this process, the map model 21] helps to capture high-level end-user's intentional requirements. The map ontology, the domain ontology and the OWL-S ontology are used to formalise the high-level end-user's intentional requirements and to specify associated generic Web Service descriptions. RDF annotations representing high-level end-user's intentional requirements and SPARQL queries formalizing generic Web Service descriptions are then grouped into rules considered as reusable fragments.

\subsubsection{Elicitation step}

Figure 1.1 shows an example of high-level end-user's intentional requirement dealing with tissue and lesion classification. It is specified with the help of the map model [21. According to 21], a map is a process model in which an ordering of intentions and strategies has been included. In our case, we focus on image processing intentions and image processing strategies. A map is a labeled directed graph with intentions as nodes and strategies as edges between intentions. An image processing intention is a goal that can be achieved by following a strategy. An intention expresses what is wanted, a state or a result 


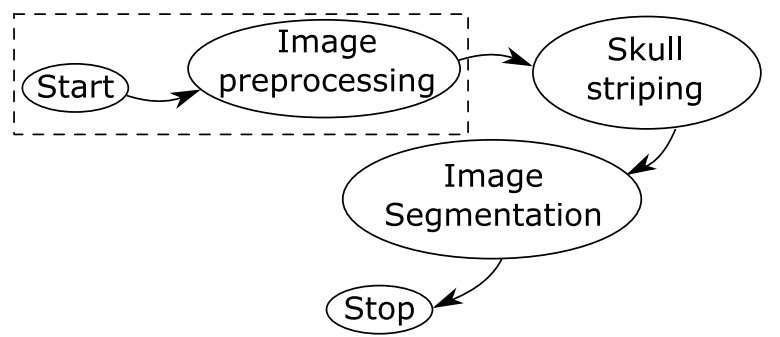

Fig. 1.1. Example of high-level end-user's intentional requirement

that is expected to be reached disregarding considerations about who, when and where. There are two distinct intentions that represent the intentions to start and to stop the process. A map consists of a number of sections each of which is a triple (source intention, target intention, strategy). A strategy characterises the flow from the source intention to the target intention and the way the target intention can be achieved. A map contains a finite number of paths from its start intention to its stop intention, each of them prescribing a way to achieve the goal of the image processing pipeline under consideration. Indeed, it is at runtime, when an intention is satisfied, that one target intention and one strategy are chosen (among all the target intention and strategies available from the current intention), depending on the context of the process at runtime.

In figure 1.1 we can see 3 main intentions: Image preprocessing, Skull striping and Image segmentation. Between the intentions, we discover strategies. Strategies define the way to pass from an intention to a next one. There can be many strategies which link up the same intentions (for instance to indicate which (kind of) algorithm is used to achieve the target intention). Indeed, in a map, each set which is made up by a source intention, a strategy and a target intention is a section of the map. An example of section has been highlighted with a doted line in figure1.1 Let's precise that a map is neither a state diagram, because there is no data structure, no object, and no assigned value, nor an activity diagram, because there is always a strong context for each section of the map: its source intention and its strategy. We can attach more information to this kind of schema (in order to help the user of the map to choose the adequate strategy, for example), but this is not the goal of this paper to fully describe the map model.

The aim of such a modeling is to capture high-level end-user's intentional requirement in order to turn them into generic Web Service description to search for available Web Services to implement the image processing pipeline under consideration. Indeed, high-level end-user's intentional requirement may need to be further refined to be transformable into generic Web Service description. For instance, in the example of figure 1.1, additional specification would be useful to understand what kinds of generic Web Service descriptions are suitable to search for Web Services implementing image preprocessing. 


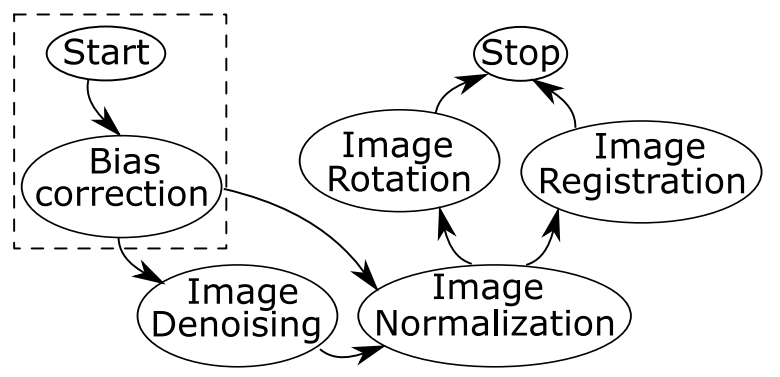

Fig. 1.2. Example of requirement refinement

Therefore, each section of a map may be refined into another map describing more in detail how to reach the target intention of the section under consideration. Figure 1.2 shows an example of map refining the section highlighted in Figure 1.1. In this map, different ways (i.e. different paths) to achieve the target preprocessing intention are provided.

At the refinement level presented in figure 1.2 it is now possible to associate generic Web Service descriptions to map sections in order to specify how to retrieve Web Service description implementing the section target intention. For instance, a query searching for Web Service descriptions which have an image as input, and an image qualified as debiased as well as a bias field as outputs aims at retrieving Web Service descriptions corresponding to the section highlighted in figure 1.2 .

\subsubsection{Formalisation step}

The second step of the authoring process is devoted to the formalisation of the intentions and strategies elicited during the previous step, as well as the generic Web Service descriptions associated to the most refined map sections. Intentions and strategies are formalised by using verbs, objects and manners from the domain ontology. Indeed, during the elicitation step, end-users think in terms of goals and means to reach goals while in the formalisation step, they try to formalise through domain concepts how to qualify goals and strategies elicited in the previous step.

To further formalise map elements, we rely on [16, 17] proposal, which has already proved to be useful to formalise goals [18, 9, 21]. According to [16, 17, an intention statement is characterised by a verb and some parameters which play specific roles with respect to the verb. Among the parameters, there is the object on which the action described by the verb is processed. We gathered the concepts and relationships of the map model and this further formalisation into an RDFS [20] ontology dedicated to the representation of intentional processes: the map ontology [5]. Intention, Strategy, Verb, Object and Manner are examples of concepts provided in this ontology. 
The mappings between the domain ontology and the map ontology are automatically created when concepts of the domain ontology are selected to formalise map content. Domain concepts are then considered as instances of Verb, Object and Manner. Let us consider again the map depicted in figure 1.2. Intention Bias correction is described by Debiasing, instance of Verb, and Image, instance of Object. With regards to strategies, up to now we only consider one strategy between a source and a target intentions. So far, we did not qualify strategies by binding them to domain concepts. In the future, we plan to extend our Web Service annotation model with quality of service (QoS) information and to qualify map strategies by QoS domain concepts considered as instances of class Manner.

By using the map and the domain ontologies, a common vocabulary is used by the different members of the community contributing to support know-how sharing and cross fertilisation.

In SATIS, we assume Web Service descriptions are expressed in OWL-S. In our current scenarios, we only use the profile and the grounding of OWL$\mathrm{S}$ as well as the input and output specifications in the process description. We enrich OWL-S description by considering Web Service OWL-S description elements (as input and output parameters for example) as instances of domain concepts. Thanks to this additional instantiation of domain concepts, it makes it possible to reason on OWL-S description element types to retrieve for instance subclasses of concepts we are interested in.

Generic Web Service descriptions are expressed as SPARQL queries among the Web Service descriptions expressed in OWL-S. The following query, where namespace process refers to the OWL-S process ontology and namespace dom refers to a domain ontology, is an example of generic Web Service description to retrieve debiasing Web Service description.

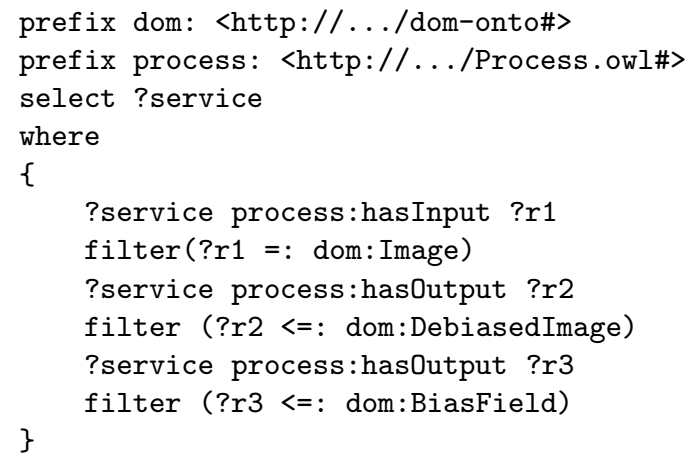

In this example, we are looking for Web Services which OWL-S description indicates that the Web Service under consideration requires a parameter instance of the Image concept from the domain ontology as input and two parameters, instances of concepts (or sub-concepts) of DebiasedImage and BiasField as output. 


\subsubsection{Fragmentation step}

In SATIS, the process consisting in retrieving Web Services descriptions from high-level end-user's intentional requirements about image processing pipelines is viewed as a set of loosely coupled fragments expressed at different levels of granularity. A fragment is an autonomous and coherent part of a search process supporting the operationalisation of part of an image processing pipeline by Web Services. Such a modular view of the process aiming at retrieving Web Service descriptions from high-level end-user's intentional requirements favours their adaptation and extension. Moreover, this view permits to reuse fragments authored to deal with a specific high-level end-user's image processing pipeline in the building of other pipelines.

The fragment body captures guidelines that can be considered as autonomous and reusable. The fragment signature captures the reuse context in which the fragment can be applied.

For us, a guideline embodies know-how about how to achieve an intention in a given situation. We distinguish two types of guidelines: intentional and operational guidelines. Intentional guidelines capture high-level end-user's intentional requirements which have to be refined into more specific requirements. Operational guidelines capture generic Web Service description.

Map formalisations and SPARQL queries respectively constitute the body of intentional and operational reusable fragments. The fragment signature characterises the fragment content and let the other members of the community understand in which situation the fragment may be useful. A fragment signature is specified by a map section. The target intention of the section indicates the goal of the reusable fragment and the source intention as well as the strategy specify the reuse situation in which the fragment is suitable. The section highlighted in figure 1.1 is an example of signature for an intentional fragment which body is the map presented in figure 1.2. The section highlighted in figure 1.2 is an example of signature for an operational fragment which body is the query presented in section 1.3.2

Indeed in SATIS, fragments are implemented by backward chaining rules, which conclusions represent signatures of fragments and which premises represent bodies of fragments (either operational or intentional guidelines). We call a rule concrete or abstract depending on whether its premise encapsulates operational or intentional guidelines.

These rules are implemented as SPARQL construct queries. The CONSTRUCT part is interpreted as the head of the rule, the consequent that is proved. The WHERE part is interpreted as the body, the condition that makes the head proved. When considered recursively, a set of SPARQL construct queries can be seen as a set of rules processed in backward chaining.

The following rule, where namespace map refers to the map ontology, namespace process refers to the OWL-S ontology and namespace dom refers to a domain ontology, is an example of concrete rule implementing an operational fragment aiming at retrieving debiasing Web Services. 




In the WHERE part of the rule, we recognise the query previously presented in this paper. In the CONSTRUCT part of the rule, a graph pattern corresponding to the map section to build if Web Service descriptions are found in the community Web Services registry is specified. This graph pattern specifies the fact that no specific strategy and no specific source intention is required to achieve the target intention (concepts map: AnyParameter, map: AnyObject and map:AnyVerb are used in the specification). It also indicates that target intention is formalised by the object dom: Image and the verb dom:Debiasing. The retrieved Web Service descriptions are associated to the newly built map section through the hasResource property.

Thanks to SATIS three steps authoring process, high-level end-user's intentional requirements are capitalised inside the community semantic memory in order to be reused during the rendering process that will be detailed in the following section. 


\subsection{SATIS rendering process}

The rendering step is supported by backward chaining among rules and matching with the Web Service descriptions. We rely on a semantic engine for both backward chaining on the SATIS knowledge base of rules implementing the reusable fragments and matching with the knowledge base of OWL-S Web Service descriptions. During the rendering step, high-level end-user's intentional requirements are dynamically created when needed all along the backward chaining process, as temporarily subgoals, until Web Service descriptions are found to match all the sub-goals and therefore the general goal of the highlevel end-user's intentional requirement. As a result, a community member looking for solutions to operationalise an image processing pipeline will take advantage of all the rules and all the Web Service descriptions stored in the community semantic memory at the time of his/her search. This memory may evolve over the time and therefore the Web Service descriptions retrieved by using a rule may vary as well.

Let's clarify that the result is composed of descriptions of candidate Web Services, and not by Web Services themselves. The invocation of the selected (among the candidates) Web Services is out of the scope of this work. When rendering a Web Service descriptions search process, a set of candidate Web Services (alternatives) is associated to each goal or subgoal elicited during the specification of the image processing pipeline. So, the result of the rendering is a sequence of sets of candidate Web Services. But as the formalism we choose to model image processing pipeline, the map model [21, allows to specify several way to achieve an intention, the result of the rendering step may be composed of several sequences of sets of candidate Web Services.

\subsection{Improving collaboration among community members}

One of the main objectives of SATIS is to support neuroscientists when looking for Web Services to operationalise their image processing pipeline. In this section we will first discuss the role of the different actors involved in the neuroscience community and then describe the different means we provide to support neuroscientists tasks.

Three core actors are identified in our framework: the service designer, the process modeling expert and the domain expert. In a neuroscientists community, computer scientists play the roles of service designer and process modeling expert while neuroscientists play the role of domain expert.

The service designer is in charge of promoting the Web Services available in the community Web Service registry. Therefore, when s/he wants to advertise a new kind of Web Service in the neuroscientists community, in addition to adding the Web Service description in the community Web Service registry, $\mathrm{s} /$ he writes a generic Web Service description and associates to it high-level end-user's intentional requirements to promote the services s/he is in charge 
from the end-user's point of view (that is to say in a non computer scientists language, as OWL-S is). The service designer is in charge of authoring atomic reusable fragments.

The process modeling expert is in charge of populating the community semantic memory with reusable fragments to help domain experts to (i) specify the image processing pipelines for which they are looking for Web Services and (ii) search for Web Service descriptions to operationalise the image processing pipelines they are interested in. Indeed, s/he provides reusable fragments useful in different image processing pipelines. Basic processes, as for instance intensity corrections, common to several image analysis pipelines, are examples of such basic fragments. Therefore, $\mathrm{s} /$ he may look at the fragments provided by the service designer with the aim of aggregating some of them into basic image processing pipelines. For instance, if Image debiasing, Image denoising, Image normalisation and Image registration Web Service descriptions are provided in the community Web Service registry (and associated fragments provided in the community semantic memory) at some point, the process modeling expert may put them together into a basic Image preprocessing pipeline. S/he may also identify recurrent needs when supporting domain experts in their authoring task and therefore provide adequate basic fragments for image processing pipelines. The process modeling expert may therefore write abstract rules. If concrete rules about generic Web Service descriptions corresponding to image processing subgoals are already available, the process modeling expert only writes the abstract rules. Otherwise, $\mathrm{s} /$ he is also in charge of writing the associated concrete rules.

Finally, the domain expert (or final user) is searching for Web Service descriptions to operationalise an image processing pipeline $\mathrm{s} / \mathrm{he}$ is interested in. Therefore, $\mathrm{s} /$ he may first look in the community semantic memory if some existing rules already deal with the main intention $\mathrm{s} /$ he is interested in. If another member of the community already authored an image processing pipeline achieving the same high-level goal, s/he may reuse it as is. The goal under consideration may also be covered by a larger image processing pipeline specified through a set of rules already stored in the community semantic memory and corresponds to one of the subgoals of the larger pipeline. In this case also, existing rules can be reused as is and the rendering step to operationalise the image processing pipeline under consideration performed on the current semantic community memory content. If no high-level end-user's intentional requirements are already available, the domain expert specifies the image processing pipeline under consideration with the help of the process modeling expert. Indeed, abstract rules have to be written. Then, for each subsection identified in the high-level abstract rule, the domain expert may search for existing rules supporting their operationalisation. If it is the case, then $\mathrm{s} /$ he can decide to rely on them and stop the authoring process. Otherwise, s/he may prefer to provide his/her own way to operationalise the subgoals. By doing so, the domain expert enriches the semantic community memory with alternative ways to operationalise already registered goals. This 
will result in enriching the operationalisation means of the image processing pipelines already formalised into rules stored in the semantic community repository. In fact, when someone else looking for the subgoals under consideration will perform a rendering process, if his/her image processing pipeline relies on the achievement of a target intention for which a new operationalisation means has been provided, the backward chaining engine will exploit the rules previously stored in the semantic community repository as well as the new ones, increasing the number of ways to find suitable Web Service descriptions. Each time the domain expert, with the help of the process modeling expert, decides to provide new ways to operationalise a map section, s/he has to select the right level of specification of the fragment signature, in order to allow the reuse of the fragment under construction outside of the scope of the image processing pipeline under consideration.

From a more general point of view, domain and process modeling experts mainly provide intentional fragments: Domain experts focus on high-level intentional fragments, close to the image processing pipelines they want to operationalise. Process modeling experts focus on low level intentional fragments, that is to say fragments operationalising basic image processing pipelines. And service designers mainly focus on providing operational fragments to promote existing Web Services. But domain and process modeling experts may also provide operational fragments to specify their requirements in term of services. And the service designers may also provide intentional fragments in order to show examples of use of available Web Services inside the scope of more complex examples of image processing pipelines. By relying on a rulebased specification to retrieve Web Service descriptions and by providing distinct and dedicated modeling techniques to both service designers and service final-users as well as mapping mechanisms between them, we assist the bidirectional collaboration between neuroscientists and computer scientists inside the community.

An important objective of the SATIS project is to provide to domain experts means to better understand what are the characteristics of the available services and how to use them in the scope of the image processing pipeline they are interested in. We support this aim by several means:

- Requirements about Web Services are described in terms of intentions and strategies that is to say a vocabulary familiar to the domain expert, making the understanding of the a Web Service purpose easier to understand by domain experts.

- The SATIS approach relies on a controlled vocabulary (domain ontology) to qualify Web Services as well as requirements, this way reducing the diversity in the labeling, especially in Web Services descriptions elements.

- We propose to specify required Web Service functionalities in terms of queries (i.e. generic Web Service descriptions) instead of traditional Web Service descriptions in order to provide an abstraction level supporting 
the categorisation of available Web Services and this way an easier understanding of the content of the registry by domain experts.

- In our approach we clearly distinguish an authoring step and a rendering step:

- During the authoring step, the focus is on the elicitation of the search procedure. The domain experts think in terms of intentions and strategies (and not in terms of services). His/her search procedure is fully described, eventually with the help of the fragments already present in the community semantic memory.

- During the rendering step, it is the system (and not the domain expert) which tries to find Web Services corresponding to the requirements specified by the experts (by proving goals and sub-goals). Indeed, the experts don't need at all to know the content of the registry. A pertinent subset of it will be extracted by the system and shown to the experts.

- And finally, SATIS relies on a rule based approach which doesn't show to the domain expert the full set of rules exploited by the backward chaining engine to satisfy the user requirements. When rendering a search procedure, the domain expert only selects the intention characterizing his/her image processing pipeline and the system will search for the rules to use. A set of Web Services descriptions is given to the domain expert as result. But the complexity and the number of rules used to get the solution are hidden to the domain expert.

\subsection{Conclusion}

In this paper, we presented SATIS, a framework to derive Web Service specifications from end-user's requirements in order to operationalise business processes in the context of a specific application domain. More precisely, our framework offers the capability to capture high-level end-user's requirements in an iterative and incremental way and to turn them into queries to retrieve Web Services descriptions. The whole framework relies in reusable and combinable elements which can be shared out inside the scope of a community of users. In our approach, we adopt Web semantic languages and models as a unified framework to deal with (i) high-level end-user's intentional requirements, (ii) generic Web Service descriptions and (iii) Web Service descriptions themselves. SATIS aims at supporting collaboration among the members of a neuroscience community by contributing to both mutualisation of high-level intentional specification and cross-fertilisation of know-how about Web Services search procedures among the community members.

Future works will first focus on adapting our model to CORESE [7, 3, a semantic Web search engine including a backward chaining mechanism in order to test our approach on examples of image processing pipelines. We also plan to develop software tools in order to automate the main tedious steps, like the transformation of the map specification into SPARQL rules and to test our 
approach in the context of a neuroscientist community. We also have in mind to enrich the formalisation step by taking into account additional information in order, for instance, to derive criteria related to quality of services. Indeed, we plan to extend our Web Service annotation model with quality of service (QoS) information and to qualify map strategies by QoS domain concepts. And we will also concentrate on providing query patterns to help experts writing generic Web Service descriptions.

\section{References}

1. B. Benatallah, M.S. Hacid, C. Rey, F. Toumani, Request rewriting-based Web Service discovery. In: Fensel D, Sycara K, Mylopoulos J (eds) Proceedings of the international Semantic Web conference (ISWC 2003), Sanibel Island, FL, October 2003. Lecture notes in computer science, vol. 2870. Springer, Berlin Heidelberg NewYork, pp. 242-257.

2. L.O. Bonino da Silva Santos, L. Ferreira Pires, M.J. van Sinderen, A Goal-Based Framework for Dynamic Service Discovery and Composition. International Workshop on Architectures, Concepts and Technologies for Service Oriented Computing, July 2008, Porto, Portugal. pp. 67-78.

3. O. Corby, R. Dieng-Kuntz, C. Faron-Zucker, Querying the semantic Web with the CORESE search engine. 16th European Conference on Artificial Intelligence (ECAI/PAIS). pp. 705-709. Valencia, Spain. 2008.

4. O. Corby, R. Dieng-Kuntz, C. Faron-Zucker, F. Gandon, Searching the Semantic Web: Approximate Query Processing based on Ontologies. IEEE Intelligent Systems Journal. 21(1). 2006.

5. O. Corby, C. Faron-Zucker, I. Mirbel, Implementation of intention-driven search processes by SPARQL queries. 11th International Conference on Enterprise Information Systems (ICEIS). Milan, Italy. 2009.

6. R. Dieng, O. Corby, F. Gandon, A. Giboin, J. Golebiowska, N. Matta, M. Ribière, Knowledge management : Méthodes et outils pour la gestion des connaissances, dunod, 2005

7. Edelweiss Team of INRIA Sophia-Antipolis, CORESE: COnceptual REsource Search Engine, http://www-sop.inria.fr/edelweiss/software/corese/, 2009.

8. J.M. Gomez, M. Rico, F. Garcia-Sanchez, GODO: Goal Oriented Discovery for Semantic Web Services. 5th International Semantic Web Conference. 2006.

9. G. Guzelian, Modelisation et specification de composants réutilisables pour la conception de systèmes d'information, PhD thesis, Université Aix Marseille, 2007.

10. R.S. Kaabi, Une approche méthodologique pour la modélisation intentionnelle de services et leur opérationnalisation. PhD Thesis, Université Paris I Sorbonne. February 2007.

11. L. Li and I. Horrocks, A Software Framework for Matchmaking Based on Semantic Web Technology. International Journal of Electronic Commerce. Volume 8, Issue 4, Pages 39-60, 2004.

12. D. Martin M. burnstein, D. McDermott, S. McIlraith, M. Paolucci, K. Sycara, D.L. McGuinness, E. Sirin and N. Srinivasan, Bringing Semantics to Web Services with $O W L-S$. World Wide Web (2007). 10:243-277.

13. S. Nurcan, M.H. Edme, Intention-driven modeling for flexible workflow applications. Journal of Software Process: Improvement and Practice. 10(4). 2005. 
14. OWL-S Coalition, OWL-S Specification. http://www.ai.sri.com/daml/services/owl$\mathrm{s} / 1.2$.

15. M. Paolucci, T. Kawamura, T.R. Payne and K. Sycara, Semantic Matching of Web Services Capabilities. In First Int. Semantic Web Conference, Sardinia, Italy, June 2002.

16. N. Prat, Goal formalization and classification for requirements engineering, Third International Workshop on Requirements Engineering: Foundations of Software Quality, (REFSQ), 1997, Barcelona, Spain.

17. N. Prat, Réutilisation de la trace par apprentissage dans un environnement pour l'ingénierie des processus, PhdThesis, Université Paris I - Sorbonne, 1999.

18. J. Ralyt, Ingénierie des méthodes à base de composants, $\mathrm{PhD}$ thesis, Université Paris I - Sorbonne, 2001.

19. W3C, RDF: Resource Description Framework, http://www.w3.org/RDF/, 2009.

20. W3C, RDF Vocabulary Description Language 1.0: RDF Schema, http://www.w3.org/TR/rdf-schema/, 2004.

21. C. Rolland, Conceptual Modelling in Information Systems Engineering. Springer-Verlag, 2007.

22. W3C, SPARQL Query Language for RDF, W3C Recommendation, http://www.w3.org/TR/rdf-sparql-query/, 2008.

23. M. Stollberg, B. Norton A Refined Goal Model for Semantic Web Services. Second International Conference on Internet and Web Applications and Services (ICIW'07), 2007.

24. M. Vukovic, P. Robinson GoalMorph: Partial Goal Satisfaction for Flexible Service Composition. International Conference on Next Generation Web Services Practices. 2005.

25. K. Zhang, Q. Li, Q. Sui A Goal-driven Approach of Service Composition for Pervasive Computing. 1st International Symposium on Pervasive Computing and Applications, pp. 593-598, August 2006. 